\title{
COMPREHENSIVE STUDY OF ORAL HYGIENE STATUS IN 40-50 YEAR-OLD WOMEN RANGES USING DRUG PRODUCT TO INCREASE RESISTANCE OF DENTAL HARD TISSUES.
}

\author{
Barylo O.S. ${ }^{1}$, Agafonov K.V. ${ }^{2}$, Kirichek O.V. ${ }^{3}$, Furman R.L. ${ }^{\star 4}$ \\ ${ }^{1}$ Department of Surgical Dentistry and Maxillofacial Surgery, Vinnytsia National Medical University named after MI Pirogov, \\ Pirogova st., 56, Vinnitsa, Ukraine \\ ${ }^{2}$ Department Dentistry, Odessa Medical Institute of the International Humanitarian University Department of General dentistry, \\ Odessa, Ukraine \\ ${ }^{3}$ Department Dentistry, Odessa Medical Institute of the International Humanitarian University Department of General dentistry, \\ Odessa, Ukraine \\ ${ }_{4}^{4}$ Department of Surgical Dentistry and Maxillofacial Surgery, Vinnytsia National Medical University named after MI Pirogov, \\ Pirogova st., 56, Vinnitsa, Ukraine
}

DOI: https://doi.org/10.15520/ijmhs.v9i3.2483

Accepted 9 Mar 2019; Received 1 Feb 2019; Publish Online 22 Mar 2019

\section{Reviewed By: Dr.}

Daniel V.

Department: Medical

\begin{abstract}
Resistance of tooth enamel to aggressive effect of metabolic by-products of oral cavity microorganisms is provided by fluoroapatites of enamel surface layers. The patients were divided into two groups: control group (30 females) and experimental (observation) group (30 females). In control group, the traditional method of caries prevention was used, which included professional teeth cleaning, mouthwash with physiological solution $(0.9 \%)$ of sodium chloride after meals, hygienic teeth brushing by a standard method twice a day. Females of experimental group underwent professional teeth cleaning, treatment of teeth with the drug product containing sodium fluoride to increase the resistance of hard tissues of teeth, silver nitrate and decamethoxin, rinsing with standardized decamethoxin solution. Various indices are used in modern dentistry to evaluate oral hygiene status. Index estimation provides quantitative characteristics of static status of oral hygiene. Their values enable to suggest the course of inflammatory-destructive or dystrophic process and its severity, to evaluate treatment efficacy and quality of regular check-up of a particular patient or dispensary cases. The study demonstrated significant positive clinical efficacy of the drug product used to increase resistance of dental hard tissues. In particular, patients using the pharmaceutical preparation to improve the resistance of dental hard tissues, had significantly lower values of Fedorov-Volodkina's Index, Greene-Vermillion Index (Oral Hygiene Index- Simplified), Patient Hygiene Performance (PHP) Index as compared to the control group.
\end{abstract}

Key words: Resistance of tooth-silver nitrate-decamethoxin

\section{INTRODUCTION}

In spite of the fact that certain progress has been achieved in prevention of dental carious in Ukraine, its high prevalence and intensity is still registered, particularly among middle-aged women with estrogen imbalance, being an ur-

${ }^{\star}$ Corresponding author: furmanruslan1977@gmail.com. gent problem in modern dentistry. According to literature data, the prevalence of permanent teeth caries in 40-50 yearold women ranges from 72.7 to $94.3 \%$ with the intensity of damage 2.5 - 4.7 teeth $[3,4]$.

The problem of etiology of dental caries is recognized to be solved by the majority of investigators. Dental plaque microflora is considered to be the major cause of caries. Microbial (infectious) theory of origin of dental caries has 
been comprehensively and consistently proved both experimentally and clinically. Other theories and concepts seem to reflect the significance of definite links in pathogenesis of the disease. Caries develops as a result of pathogenic effect of microbial plaque on tooth enamel in places with favourable conditions for retention and accumulation of plaque on teeth surface $[2,8]$.

Resistance of tooth enamel to aggressive effect of metabolic by-products of oral cavity microorganisms is provided by fluoroapatites of e namel s urface layers. According to $\mathrm{WHO}$, the most effective a gent p reventing t he development of caries is fluoride, especially its topical application. Because of that, it is widely present in toothpastes, mouthwashes, mouth rinses, dental varnishes, gels, and sealants $[1,7]$.

Demineralization of bones and teeth contributes to decreased resistance of dental hard tissues as well. The process of calcium metabolism is influenced by a $n$ umber of hormones, their concentration changing in various periods of women's life. In women aged 40-50, natural deficiency of blood estrogen level develops due to age-related changes in gonads. The mechanisms of influence of s ex h ormones on bone tissue are critically important but not studied completely. However, after detection of specific o steoblast receptors to estrogens, androgens, growth hormone and thyroid hormones, it became apparent that spongy substance of bone tissue is a particular kind of target organ for sex hormones. Estrogens exert the most significant e ffect on bone and mineral metabolism, since they activate osteoblasts, inhibit interleukin production, promote inhibition of bone resorption, decrease bone tissue susceptibility to resorptive effect of $\mathrm{p}$ arathyroid $\mathrm{h}$ ormone, i ncrease $\mathrm{b}$ one $\mathrm{t}$ issue sensitivity to vitamin D3, enhance calcitonin synthesis, regulate the processes of calcium absorption and secretion, activate apoptosis of osteoclasts. Decrease of estrogen level leads to accelerated bone metabolism and loss of bone substance. This significantly s lows d own the p rocesses of $b$ one tissue regeneration and dental hard tissues, leading to decreased resistance of hard tissues of teeth to the effects of aggressive factors [5, 6].

Prevention of bacterial biofilm formation on the teeth, increase of tooth enamel resistance in female patients with estrogen imbalance (requiring further study), development of new approaches to comprehensive caries prophylaxis are considered to be the urgent problems in modern dentistry.

Objective. To improve oral hygiene in female patients with estrogen imbalance and to increase their tooth enamel resistance.

\section{MATERIALS AND METHODS}

60 female patients aged 40-50 with no history of neoplasms and gonadal disorders, severe concomitant diseases (diabetes mellitus, uncompensated hypertension, uncompensated gastrointestinal diseases, etc.) were studied being under dynamic observation. The patients were divided into two groups: control group (30 females) and experimental (observation) group (30 females). In control group, the traditional method of caries prevention was used, which included professional teeth cleaning, mouthwash with physiological solution $(0.9 \%)$ of sodium chloride after meals, hygienic teeth brushing by a standard method twice a day. Females of experimentalgroup underwent professional teeth cleaning, treatment of teeth with the drug product containing sodium fluoride to increase the resistance of hard tissues of teeth, silver nitrate and decamethoxin, rinsing with standardized decamethoxin solution.

Various indices are used in modern dentistry to evaluate oral hygiene status. Index estimation provides quantitative characteristics of static status of oral hygiene. Their values enable to suggest the course of inflammatory-destructive or dystrophic process and its severity, to evaluate treatment efficacy and quality of regular check-up of a particular patient or dispensary cases.

The study was devoted to evaluation and analysis of oral hygiene indices in female patients with estrogen imbalance. The following index estimates were used:

- Fedorov-Volodkina's Index;

- Green-Vermillion Index (Oral Hygiene Index- Simplified)

- PHP (Patient Hygiene Performance) Index

Fedorov-Volodkina's Index (1971) is determined by staining labial surface of six lower frontal teeth with iodinecontaining solutions (Schiller-Pisarev solution or other iodine solution).

Quantitative estimation is done by 5 -score system:

5 - staining of all tooth crown surface;

4 - staining of $3 / 4$ of tooth crown surface;

3 - staining of $1 / 2$ of tooth crown surface;

2 - staining of $1 / 4$ of tooth crown surface;

1 - no staining of teeth.

The index value is determined by the formula:

$\mathrm{OHI}=\mathrm{y} / 6$, where $\mathrm{OHI}-$ oral hygiene index, $\mathrm{y}$ - the sum vahofes of 6 teeth.

Oral hygiene index is interpreted in the following way:

1.1-1.5 scores - good oral hygiene level;

1.6-2.0 scores - satisfactory;

2.1-2.5 scores - unsatisfactory;

2.6-3.4 scores - bad;

3.5-5.0 scores - very bad.

Green-Vermillion Index (1964) (Oral Hygiene

Index- Simplified)

To determine Oral Hygiene Index- Simplified, the vestibular surfaces of 16, 11, 26, 31 teeth, and lingual surfaces of 36 and 46 teeth are stained with Schiller-Pisarev solution or other iodine solution. The amount of debrisDebris-index (DI), and calculus - Calculus-index (C), found on study tooth surfaces are scored (Table 1).

Patient Hygiene Performance (PHP) Index

For qualitative estimation of dental plaque, the following 6 teeth are stained:

$16,26,11,31$ - vestibular surfaces

36. 46 - lingual surfaces. 
Table 1. Criteria for scoring Green-Vermillion Index

\begin{tabular}{lll}
\hline Score Debris-index & Calculus-index (C) \\
0 & $\begin{array}{l}\text { No debris } \\
\text { present }\end{array}$ & No calculus present \\
1 & $\begin{array}{l}\text { Soft debris } \\
\text { covering one } \\
\text { third of tooth }\end{array}$ & tooth surface \\
& $\begin{array}{l}\text { surface } \\
\text { Soft debris } \\
\text { covering two } \\
\text { thirds of tooth }\end{array}$ & $\begin{array}{l}\text { Supragingival calculus covering two } \\
\text { thirds of tooth surface; subgingival } \\
\text { calculus in the form of separate }\end{array}$ \\
3 & $\begin{array}{l}\text { surface } \\
\text { Soft debris } \\
\text { covering more } \\
\text { than two thirds } \\
\text { of tooth surface }\end{array}$ & $\begin{array}{l}\text { Supragingival calculus covering two } \\
\text { thirds of exposed tooth surface } \\
\text { and/or subgingival calculus covering } \\
\text { pre-cervical part of the tooth }\end{array}$ \\
\hline
\end{tabular}

If the index tooth is absent, the neighboring tooth of the same group is examined. Artificial crowns and parts of fixed dentures are examined in the same way as natural ones.

The surface of each tooth is divided into 5 areas:

1 -medial;

2 - distal;

3 - medial-occlusive;

4- central;

5 - medial-precervical.

The scores and criteria of debris estimates are:

0 - no staining on individual area;

2 - definite staining on individual area.

PHP index is calculated as the total of the score for each surface area. For instance, if the staining is observed on one surface area - distal one, the score of debris is 1 . If the staining is observed on three surface areas - medial, distal and medial-precervical, the score of debris is $1+1+1=3$. If the staining is observed on four surface areas - medial, distal, medial-precervical and central, the score of debris is $1+1+1+1=4$. Thus, the calculation formula is:

$\mathrm{PHP}=$ total of the scores for all teeth divided by the number of teeth examined.

PHP index is interpreted in the following way:

0 scores - excellent oral hygiene;

0.1-0.6 - good oral hygiene;

0.7-1.6 - satisfactory oral hygiene;

$>1.7$ - unsatisfactory oral hygiene.

Oral hygiene status was evaluated for three times during the study period:

- first evaluation - at initial presentation of female patients after professional cleaning of teeth:

- second evaluation - on the 7th day of observation;

- third evaluation - on the 28th day of observation.

\section{RESULTS}

The results obtained during the study of oral hygiene status in female patients with estrogen imbalance using the pharmaceutical to increase resistance of hard tooth tissues were analyzed.

After calculation of oral hygiene indices the following results were obtained.

In control group Fedorov-Volodkina's Index was 1on the first day, $1.85 \pm 0.24$-on the 7 th day, $2.39 \pm 0.18$ - on the 28 th day. In experimental group those values were $1.05 \pm 0.10$, $1.19 \pm 0.21$ and $1.28 \pm 0.09$, respectively. (Table 2)

Table 2. Fedorov-Volodkina's Index

\begin{tabular}{|c|c|c|c|}
\hline & 1st day & 7th day & 28th day \\
\hline $\begin{array}{l}\text { Control } \\
\text { group }\end{array}$ & 1 & $1.85 \pm 0.24$ & $2.39 \pm 0.18$ \\
\hline $\begin{array}{l}\text { Experimen- } \\
\text { tal } \\
\text { group }\end{array}$ & $\begin{array}{l}1.05 \pm 0.10 \\
\mathrm{p} 1>0.05\end{array}$ & $\begin{array}{l}1.19 \pm 0.21 \\
\mathrm{p} 2<0.05\end{array}$ & $\begin{array}{l}1.28 \pm 0.09 \\
\text { p3<0.05 }\end{array}$ \\
\hline
\end{tabular}

$\mathrm{p}_{1}$ - significance of difference betweenexperimental group and control group on 1st day;

$\mathrm{p}_{2}$ - significance of difference betweenexperimental group and control group on 7 th day;

$\mathrm{p}_{3}$ - significance of difference between experimentalgroup and control group on 28th day; ( the same notes relate to the subsequent tables)

In control group Green-Vermillion Index (Oral Hygiene Index- Simplified) was 0 on the first day, $1.16 \pm 0.17-$ on the 7 th day, $1.87 \pm 0.22-$ on the 28 th day. In experimentalgroup those values were $0.03 \pm 0.09,0.22 \pm 0.11$ and $0.27 \pm 0.21$, respectively (Table 3 ).

Table 3. Green-Vermillion Index (Oral Hygiene IndexSimplified)

\begin{tabular}{llll}
\hline & 1st day & 7 th day & 28th day \\
$\begin{array}{l}\text { Control } \\
\text { group }\end{array}$ & 0 & $1.16 \pm 0.17$ & $1.87 \pm 0.22$ \\
$\begin{array}{l}\text { Experimen- } \\
\text { tal } \\
\text { group }\end{array}$ & $\begin{array}{l}0.03 \pm 0.02 \\
\mathrm{p} 1>0.05\end{array}$ & $\begin{array}{l}0.22 \pm 0.11 \\
\mathrm{p} 2<0.05\end{array}$ & $\begin{array}{l}0.27 \pm 0.21 \\
\mathrm{p} 3<0.05\end{array}$ \\
\hline
\end{tabular}

Patient Hygiene Performance (PHP) Index in control group was 0 on the first day, $1.59 \pm 0.21$ - on the 7 th day, $2.04 \pm 0.21$ - on the 28 th day. In experimental group those values were $0.04 \pm 0.02,0.29 \pm 0.06$ and $0.42 \pm 0.16$, respectively (Table 4).

Table 4. Patient Hygiene Performance (PHP) Index

\begin{tabular}{llll}
\hline & 1st day & $\begin{array}{l}\text { 7th day } \\
1.59 \pm 0.21\end{array}$ & $\begin{array}{l}28 \text { th day } \\
2.04 \pm 0.21\end{array}$ \\
$\begin{array}{l}\text { Control } \\
\text { group }\end{array}$ & 0 & & \\
$\begin{array}{l}\text { Experimen- } \\
\text { tal } \\
\text { group }\end{array}$ & $0.04 \pm 0.02$ & $0.29 \pm 0.06$ & $0.42 \pm 0.16$ \\
p1 $>0.05$ & $\mathrm{p} 2<0.05$ & $\mathrm{p} 3<0.05$ \\
\hline
\end{tabular}

\section{CONCLUSIONS}

The study demonstrated significant positive clinical efficacy of the drug product used to increase resistance of dental 
hard tissues. In particular, patients using the pharmaceutical preparation to improve the resistance of dental hard tissues, had significantly lower values of Fedorov-Volodkina's Index, Greene-Vermillion Index(Oral Hygiene Index- Simplified), Patient Hygiene Performance (PHP) Index as compared to the control group. This is indicative of the fact that the pharmaceutical agent intended to improve the resistance of dental hard tissues, promotes decreased adhesion of dental debris and plaque formation, thus leading to decreased aggressive influence of microorganisms on hard tissues of the teeth.

\section{REFERENCES}

[1] Beloklitskaya GF, Pavlenko EM, Rudenko AV. Study of bactericidal activity of argentum preparations against pathogens of inflammatory processes in periodontal tissues. Modern Dentistry. 2014;5:18-22.

[2] Bezvushko EV. Prevention of caries of first permanent molars in children on individual level. Dentistry News. 2009;2:67-71.

[3] Gadzhula NG. Individual prophylaxis of dental caries in women during pregnancy and lactation: extended abstract of Cand. Med. Sci. Dissertation: spec.14.01.22 "Dentistry" Lviv National D.Halytsky Memorial. Medical University; 2009. Lviv. 20p.

[4] Gromova SN, Rumyantsev VA. Dentistry. 2012;2:16-19.

[5] Kulygina VM, Pylypiuk OYu, Komnatsky BYu. Clinical Dentistry. 2016;1:63-67.

[6] F KL. The results of study of electrolyte contents, enzyme activity of alkaline and acid phosphatase in oral fluid of adolescents after treatment and prevention of multiple dental caries. Ukrainian Almanac of Dentistry. 2012;5:31-34.

[7] Clinical and experimental reasoning for ways and methods of increasing resistance to caries of permanent tooth enamel with various mineralization level: extended abstract of Dr. Med. Sci. Dissertation: spec. 14.01.22 "Dentistry". Ministry. 2017;Kyiv. 31p.

[8] Shatylo P, V T. Professional Oral Hygiene: Study guide. vol. 88. Zhytomyr: Polissya; 2008. Illustrative photos.

\section{AUTHOR BIOGRAPHY}

Barylo O.S. Department of Surgical Dentistry and Maxillofacial Surgery, Vinnytsia National Medical University named after MI Pirogov, Pirogova st., 56, Vinnitsa, Ukraine

Agafonov K.V. Department Dentistry, Odessa Medical Institute of the International Humanitarian University Department of General dentistry, Odessa, Ukraine

Kirichek O.V. Department Dentistry, Odessa Medical Institute of the International Humanitarian University Department of General dentistry, Odessa, Ukraine

Furman R.L. Department of Surgical Dentistry and Maxillofacial Surgery, Vinnytsia National Medical University named after MI Pirogov, Pirogova st., 56, Vinnitsa, Ukraine 